\title{
miR-23b-3p promotes the apoptosis and inhibits the proliferation and invasion of osteosarcoma cells by targeting SIX1
}

\author{
HUA LIU $^{1 *}$, WEI WEI ${ }^{2 *}$, XIAOJIAN WANG ${ }^{1}$, XIAOJUN GUAN $^{1}$, QINGQING CHEN $^{1}$, \\ ZHONGJIN PU ${ }^{3}, \mathrm{XUDONG} \mathrm{XU}^{1}$ and AICHUN WEI ${ }^{1}$ \\ ${ }^{1}$ Department of Orthopedics, Haian Hospital of Traditional Chinese Medicine, Haian, Jiangsu 226600; \\ ${ }^{2}$ Department of Orthopedics, Jiangsu Provincial Hospital of Traditional Chinese Medicine, Nanjing, Jiangsu 210001; \\ ${ }^{3}$ Department of Tumor, Haian Hospital of Traditional Chinese Medicine, Haian, Jiangsu 226600, P.R. China
}

Received November 10, 2017; Accepted September 4, 2018

DOI: $10.3892 / \mathrm{mmr} .2018 .9611$

\begin{abstract}
Osteosarcoma (OS) is the most common primary malignant bone tumor and the third most common cancer that occurs during childhood and adolescence. Increasing evidence has suggested that microRNA (miR)-23b-3p has an important role in OS tumorigenesis; however, the underlying molecular mechanisms remain unknown. The aim of the present study was to investigate the expression levels of miR-23b-3p and sine oculis homeobox homolog 1 (SIX1) in OS tissues and cell lines (MG-63, SaOS-2 and U2OS), as well as to observe the effects of miR-23b-3p on U2OS cell viability, cell cycle, apoptosis and invasive ability. The results revealed that the expression levels of miR-23b-3p were significantly decreased in OS tissues and cell lines compared with tumor-adjacent normal tissues and a non-cancerous human fetal osteoblastic cell line (hFOB1.19). To investigate the underlying mechanisms of miR-23b-3p in OS tumorigenesis and progression, human U2OS cell lines over- or under expressing miR-23b-3p were established. The effects of miR-23b-3p on U2OS cell viability, cell cycle, apoptosis and invasion properties were determined by performing Cell Counting Kit-8, flow cytometry and Transwell invasion assays. miR-23b-3p was revealed to suppress cell viability, proliferation and invasion, and to enhance the levels of cell apoptosis. Furthermore, SIX1 mRNA and protein expression levels in OS tissues and cell lines were significantly upregulated when compared with tumor-adjacent normal tissues and hFOB 1.19 cells, which suggested that SIX1 expression levels may be inversely associated with miR-23b-3p levels in OS. Luciferase reporter system analysis demonstrated that miR-23b-3p binds
\end{abstract}

Correspondence to: Dr Xiaojian Wang, Department of Orthopedics, Haian Hospital of Traditional Chinese Medicine, 55 Ninghai Middle Road, Haian, Jiangsu 226600, P.R. China

E-mail: liuhua_dr1979@163.com

*Contributed equally

Key words: microRNA-23b-3p, osteosarcoma, sine oculis homeobox homolog 1, apoptosis, invasion to the SIX1 3'-untranslated region. miR-23b-3p downregulation contributed to SIX1 upregulation, which facilitated the potentiation of cyclin D1 and vascular endothelial growth factor-C expression levels, as well as the inhibition of caspase-3 expression. Collectively, these results suggested that miR-23b-3p is downregulated and SIX1 is upregulated in OS cells, and that miR-23b-3p inhibition may suppress the proliferation and invasion of OS cells, and contribute to cell apoptosis via negative regulation of SIX1. miR-23b-3p/SIX1 may therefore represent a potential target for the treatment of OS.

\section{Introduction}

Osteosarcoma (OS) represents the most common primary malignant bone tumor, and most frequently occurs in individuals $<20$ years of age (1). Pulmonary metastases occurring during OS account for nearly all associated cases of mortality $(2,3)$. The 5 year survival rate for patients with localized OS remains at $60-70 \%$ when receiving multimodality therapy, including surgery and radio- and chemotherapy (4). By contrast, the 5 year survival rate for patients with aggressive metastases is only $10-30 \%$, thereby indicating an unsatisfactory response rate to multimodality therapy (5). However, as the mechanisms underlying the regulation of OS progression have not yet been clearly characterized, it remains difficult for currently available therapeutic strategies to effectively treat OS (6). Therefore, previous studies have focused on broadening the understanding of biological targets associated with OS cell malignant biological behavior, which has important theoretical and clinical significance $(7,8)$.

MicroRNAs (miRs/miRNAs) are comprised of short non-coding RNA with a length of 22 nucleotides that typically inhibit the translation and stability of mRNAs, as well as regulate genes involved in cellular processes, including inflammation, apoptosis, cell-cycle, invasion and migration (9-12). As post-transcriptional regulators of developmental processes, miRNAs have an important role in almost all biological processes, and aberrant miRNA expression is associated with numerous diseases, including cancer (13). Emerging evidence has revealed that miRNAs are involved in human carcinogenesis by functioning as tumor suppressors or oncogenes, and thus have prognostic value for patients with cancer (14). 
In addition, previous studies have also demonstrated that the expression of miR-23 is dysregulated in angiogenesis, coronary artery disease, immune responses and cancer (15-19); the dysregulation of miR-23 is associated with numerous of cancer (20-22).

The miR-23 protein family has two members: mir-23a and mir-23b (23). Cai et al (20) demonstrated that the levels of miR-23a were decreased in prostate cancer and that low levels of miR-23a were associated with poor prognosis. Furthermore, the expression of miR-23a has been demonstrated to be decreased in OS due to hypermethylation of its promoter (21). Aghaee-Bakhtiari et al (24) further demonstrated that miR-23a and miR-23b were significantly downregulated in prostate cancer, whereas their overexpression in prostate cell lines was revealed to decrease interleukin-6R expression, which has an important role in the mitogen-activated protein kinase (MAPK) and Janus kinase/signal transducer and activator of transcription signaling pathways, and in suppressing cell proliferation and neoplastic transformation. Campos-Viguri et al (25) demonstrated that the expression of miR-23b was also downregulated via methylation of its promoter, which has been suggested to inversely regulate the expression of c-Met, zinc finger E-box binding homeobox 1 and plasminogen activator, urokinase in cervical cancer cell lines.

In addition, Begum et al (26) demonstrated that inhibition of miR-23b in non-small cell lung carcinoma (NSCLC) cell lines (H1437 and H1944) significantly decreases cell doubling time (27), which is consistent with the findings of Yan et al (27) that miR-23b may inhibit ovarian cancer tumorigenesis and progression via downregulation of cyclin G1 (27). MiR-23b-3p may also inhibit autophagy mediated by autophagy related 12 and high mobility group box 2, and sensitize gastric cancer cells to chemotherapy (28). By contrast, Jin et al (29) determined that miR-23b-3p is highly upregulated in human breast cancer, whereas knocking down miR-23b-3p significantly decreased cell proliferation and migration. Therefore, it remains unclear whether miR-23b-3p functions as a tumor suppressor or as an oncogene. Dysregulation of miR-23b-3p is currently considered to be associated with various human cancers; however, the effects of miR-23b-3p on the biological behavior of OS cells are largely unknown.

Sine oculis homeobox homolog 1 (SIX1), a developmental transcription factor, represents a member of the Six gene family, which contains six members (SIX1-6) in vertebrates (30). At present, SIX1 is the most widely studied gene from this family, a previous study demonstrating that SIX1 is associated with the development of tissues and organs (31). Previous studies have indicated that SIX1 may regulate cell growth, proliferation, differentiation, migration and apoptosis (2,32). Furthermore, overexpression of SIX1 may induce epithelial-to-mesenchymal transition (EMT) and metastasis of colorectal cancer cells, whereas SIX1 knockdown may markedly suppress cell proliferation, invasion and migration (33). Similarly, SIX1 overexpression in human breast cancer cells has been demonstrated to promote EMT and metastatic dissemination (34). Consistently, SIX1 has been revealed to be highly overexpressed in breast cancer cell lines derived from the metastatic site (35). A further study observed that SIX1 induces lymphangiogenesis and metastasis via the upregulation of vascular endothelial growth factor (VEGF)-C in mouse models of breast cancer (36). Notably, it has been suggested that nuclear factor- $\kappa \mathrm{B}$ may be involved in VEGF-C expression via the p38 MAPK signaling pathway in cancer (37-39). Furthermore, our previous study demonstrated that SIX1 is upregulated in OS cell lines when compared with the human osteoblastic cell line, hFOB1.19 (2). Preliminary analyses using online software (www.targetscan.org) suggested that SIX1 may represent a target gene of miR-23b-3p, therefore we hypothesized that miR-23b-3p may be downregulated and inversely regulate the expression of SIX1 in OS cells and thereby have an important role in the occurrence and development of OS.

The aims of the present study were to investigate the expression of miR-23b-3p and SIX1 in OS tissues and cell lines by reverse transcription-quantitative polymerase chain (RT-qPCR) and western blotting analysis, and to observe changes in the cell viability, cell cycle, apoptosis and invasive abilities of U2OS cells following enhanced and suppressed expression of miR-23b-3p. These results may provide an experimental basis for the future application of miR-23b-3p and SIX1 in the treatment of OS.

\section{Materials and methods}

Samples. The present study was granted ethical approval by the Clinical Research Ethics Committee of the Haian Hospital of Traditional Chinese Medicine (Nantong, China), and written informed consent was obtained from all patients prior to enrollment. Fresh OS tissues and matched adjacent non-tumor tissues were collected from 11 patients who underwent resection surgery between August 2011 and July 2016 (Table I). None of the included patients had undergone preoperative chemotherapy, radiotherapy, other treatment history for OS or suffered from additional inflammatory diseases. All tissue samples were immediately frozen in liquid nitrogen and then stored at $-80^{\circ} \mathrm{C}$ for subsequent experimentation.

Reagents. Cell culture reagents were obtained from Gibco; Thermo Fisher Scientific, Inc. (Waltham, MA, USA). OS cell lines (MG-63, SaOS-2 and U2OS) and a non-cancerous human fetal osteoblastic cell line (hFOB1.19) were obtained from the American Type Culture Collection (Manassas, VA, USA). The following primer sequences were purchased from Shanghai Generay Biotech Co., Ltd. (Shanghai, China): miR-23b-3p forward, 5'-CGGGCATCACAT TGCCAG G-3' and reverse, 5'-CAGCCACAAAAGAGCACAAT-3'; U6 (GI:161087014) forward, 5'-CTCGCTTCGGCAGCA CA-3', and reverse, 5'-AACGCTTCACGAATTTGCGT-3'; SIX1 (NM_005982.3) forward, 5'-TGTCCTGCGGGAGTG GTA-3', and reverse, 5'-TGATGCTGGTGGGTCTGC-3'; and $\beta$-actin (NM_001101.3) forward, 5'-GTGGACATCCGC AAAGAC-3', and reverse, 5'-AAA GGGTGTAACGCAACT AA-3'. The following stem loop sequence was also purchased from Shanghai Generay Biotech Co., Ltd.: 5'-CCTGTTGTC TCCAGCCACAAAAGAGCACAATATTTCAGGAGACAA CAGGGGTAATC-3'. A bicinchoninic acid (BCA) protein concentration assay kit, TRIzol ${ }^{\circledR}$ reagent, propidium iodide, MTT, Annexin V-fluorescein isothiocyanate (FITC) kit, Cell Counting Kit-8 (CCK-8), radioimmunoprecipitation assay (RIPA) buffer and RNase A were all purchased from Beyotime 
Table I. microRNA-23b-3p expression in osteosarcoma tissues and its association with clinical pathological factors.

\begin{tabular}{|c|c|c|c|c|}
\hline Pathological parameters & No. of patients & miR-23b-3p expression negative rate $n(\%)$ & $\chi^{2}$ & P-value \\
\hline \multicolumn{5}{|l|}{ Tissue type } \\
\hline Adjacent healthy tissue & 11 & $1(9.09)$ & 5.30 & 0.02 \\
\hline Carcinoma tissue & 11 & $10(90.91)$ & & \\
\hline \multicolumn{5}{|l|}{ Sex } \\
\hline Male & 4 & $4(100.00)$ & 0.03 & 0.86 \\
\hline Female & 7 & $6(85.71)$ & & \\
\hline \multicolumn{5}{|l|}{ Age (years old) } \\
\hline$<20$ & 9 & $8(88.89)$ & 0.01 & 0.92 \\
\hline$\geq 20$ & 2 & $2(100.00)$ & & \\
\hline \multicolumn{5}{|c|}{ Size of primary carcinoma $(\mathrm{cm})$} \\
\hline$<5$ & 5 & $4(80.00)$ & 0.06 & 0.80 \\
\hline$\geq 5$ & 6 & $6(100.00)$ & & \\
\hline \multicolumn{5}{|l|}{ Enneking stage } \\
\hline $\mathrm{I}$ & 4 & $3(75.00)$ & 0.10 & 0.95 \\
\hline II & 3 & $3(100.00)$ & & \\
\hline III & 4 & $4(100.00)$ & & \\
\hline \multicolumn{5}{|l|}{ Lung metastasis } \\
\hline Negative & 5 & $4(80.00)$ & 0.06 & 0.80 \\
\hline Positive & 6 & $6(100.00)$ & & \\
\hline
\end{tabular}

miR, microRNA.

Institute of Biotechnology (Haimen, China). Polyvinylidene fluoride (PVDF) membranes were purchased from Bio-Rad Laboratories, Inc. (Hercules, CA, USA). A Transwell chamber was obtained from Corning Costar Corp. (Cambridge, MA, USA). Rabbit anti-SIX1 (ab211359)), rabbit anti-caspase-3 (ab44976) and goat anti-VEGF-C (ab18883) polyclonal antibodies were purchased from Abcam (Cambridge, MA, USA). Mouse anti-human cyclin D1 (556470) monoclonal antibodies (BD Biosciences, San Jose, CA, USA), rabbit anti $\beta$-actin (250920) polyclonal antibodies (Abbiotec, San Diego, CA, USA), as well as horseradish peroxidase (HRP)-conjugated rabbit anti-goat IgG (81-1620), rabbit anti-mouse IgG (61-6520) and goat anti-rabbit IgG (32460) (Invitrogen; Thermo Fisher Scientific, Inc.) antibodies, were all purchased for use in the present study. Electrochemiluminescence (ECL) solution was purchased from Pierce (Thermo Fisher Scientific, Inc.).

RT-qPCR to determine SIX1 and miR-23b-3p expression levels. Total RNA was extracted from OS tissue (100 mg) or OS cells $\left(5 \times 10^{7}\right)$ using either $1 \mathrm{ml} \mathrm{TRIzol}{ }^{\circledR}$ reagent or TaqMan miRNA isolation kit (Applied Biosystems; Thermo Fisher Scientific, Inc.), according to the manufacturer's protocol. cDNA was then synthesized at $42^{\circ} \mathrm{C}$ for 45 min using the first Strand cDNA Synthesis kit (Qiagen $\mathrm{GmbH}$, Hilden, Germany), and the PCR reaction was subsequently performed using the Applied Biosystems 7500 Fast Real-Time PCR System (Applied Biosystems; Thermo Fisher Scientific, Inc.). The RT-qPCR reaction was carried out at a final volume of $25 \mu \mathrm{l}$ with the addition of $5 \mu \mathrm{l}$ cDNA, and the thermocycling conditions used were as follows: an initial denaturation at $94^{\circ} \mathrm{C}$ for $3 \mathrm{~min}$, followed by 30 cycles of denaturation at $94^{\circ} \mathrm{C}$ for $30 \mathrm{sec}$, annealing at $56^{\circ} \mathrm{C}$ for $30 \mathrm{sec}$ and extension at $72^{\circ} \mathrm{C}$ for $30 \mathrm{sec}$. Following this, the reaction mixture was incubated at $72^{\circ} \mathrm{C}$ for $10 \mathrm{~min}$. $\beta$-actin was used as an internal reference gene. TaqMan miRNA Assay and TaqMan Universal PCR Master Mix (Applied Biosystems; Thermo Fisher Scientific, Inc.) were used to detect the expression of mature miR-23b-3p in normal human osteoblasts (hFOB 1.19) and OS cell lines (MG-63, SaOS -2 and U2OS), and U6 was used as the internal reference gene. The PCR reaction conditions were as those already provided. All reactions were performed in triplicate. Relative mRNA and miRNA expression levels were calculated using the $2^{-\Delta \Delta C q}$ method (40).

Western blotting. Total protein was extracted from OS tissues and OS cell lines using RIPA buffer [150 mM NaCl, $1 \%$ NP40, $0.5 \%$ sodium deoxycholate, $0.1 \%$ sodium dodecyl sulfate, $50 \mathrm{mM}$ Tris (pH 7.9), $10 \mathrm{mM} \mathrm{NaF}$, phenylmethylsulfonyl fluoride, and $1 \mathrm{X}$ protease inhibitors (complete cocktail tablets; Roche Diagnostics GmbH, Mannheim, Germany)], and quantitative analysis of the protein concentration was subsequently performed using a BCA kit. Total protein (50 $\mu \mathrm{g}$ per lane) was separated by $12 \%$ SDS-PAGE, and then transferred to PVDF membranes. Following this, PVDF membranes were blocked with 5\% non-fat milk in Tris-buffered saline containing Tween-20 (TBST; $10 \mathrm{mM}$ Tris $\mathrm{HCl}, 150 \mathrm{mM} \mathrm{NaCl}$, and $0.1 \%$ Tween-20, $\mathrm{pH} 7.5$ ) at room temperature for $1 \mathrm{~h}$. The PVDF membranes were subsequently incubated at $4^{\circ} \mathrm{C}$ overnight with the following primary antibodies: Rabbit anti-SIX1 polyclonal antibodies (1:1,000), rabbit anti-caspase-3 polyclonal antibodies 

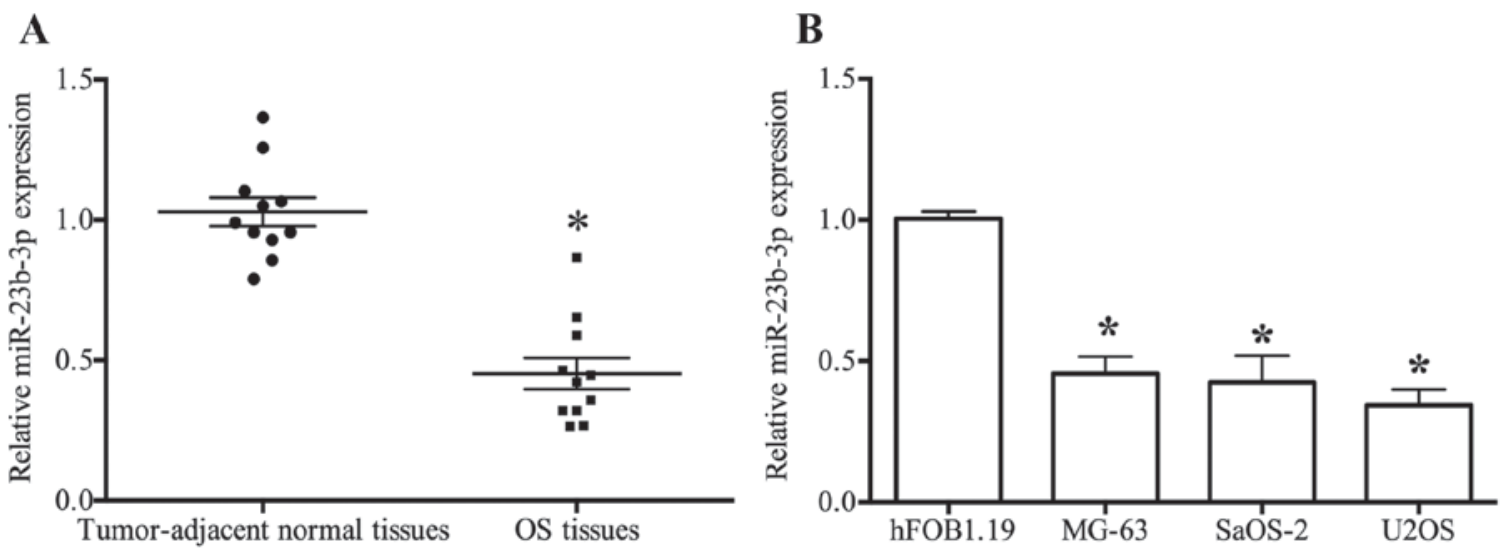

Figure 1. Relative expression of miR-23b-3p in OS tissues and cell lines. (A) The relative expression of miR-23b-3p in OS tissues. "P<0.05 vs. normal tumor-adjacent tissues. (B) The relative expression of miR-23b-3p in OS cell lines. "P<0.05 vs. hFOB 1.19 cells. miR, microRNA; OS, osteosarcoma.
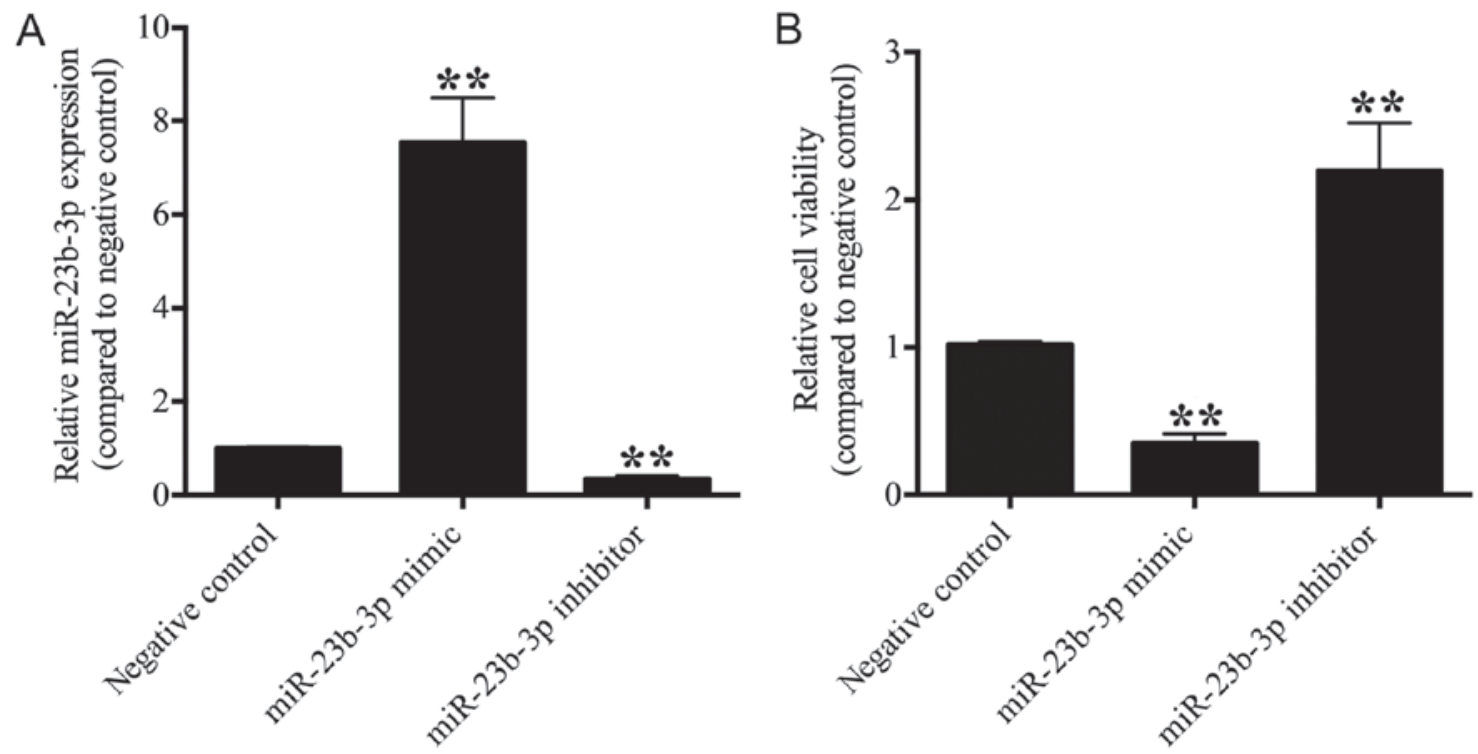

Figure 2. (A) Histograms presenting the relative expression of miR-23b-3p and (B) the relative cell viabilities of U2OS cells. ${ }^{* *} \mathrm{P}<0.01$ vs. negative control. miR, microRNA.

(1:2,000), goat anti-VEGF-C polyclonal antibodies $(1: 2,000)$, monoclonal mouse anti-cyclin D1 antibodies $(1: 2,000)$ and rabbit anti $\beta$-actin antibodies $(1: 2,000)$. Following this, PVDF membranes were washed with TBST and then incubated with HRP-conjugated secondary antibodies $(1: 2,000)$ at $37^{\circ} \mathrm{C}$ for $1 \mathrm{~h}$. Finally, PVDF membranes were washed again with TBST and the bands were subsequently visualized using ECL reagent. Densitometric analysis was performed using QuantityOne software version 4.62 (Bio-Rad Laboratories, Inc.).

Cell culture. Human OS MG-63, SaOS-2 and U2OS cells were cultured in RPMI-1640 supplemented with $10 \%$ fetal bovine serum (FBS) (Gibco; Thermo Fisher Scientific, Inc.) and $1 \%$ penicillin/streptomycin at $37^{\circ} \mathrm{C}$ in a humidified atmosphere containing $5 \% \mathrm{CO}_{2}$. hFOB1.19 cells were cultured in Dulbecco's modified Eagle medium F-12 (Gibco; Thermo Fisher Scientific, Inc.) containing $0.5 \mathrm{mM}$ sodium pyruvate, $2.5 \mathrm{mM}$ L-glutamine, $15 \mathrm{mM}$ HEPES, $1 \%$ penicillin/streptomycin and 10\% FBS (Invitrogen; Thermo Fisher Scientific,
Inc.) at $34^{\circ} \mathrm{C}$ in a humidified atmosphere containing $5 \% \mathrm{CO}_{2}$. Inverted microscopes were used to observe the growth rate of OS cells. When cells reached $70-80 \%$ confluence, they were digested using $0.25 \%$ trypsin. Cells in the logarithmic growth phase were isolated for further experimentation.

Cell treatment. U2OS cells were selected for the performance of subsequent experiments. Normal cultured U2OS cells $\left(3 \times 10^{5}\right)$ were inoculated into 6-well plates. Following cell adherence, miR-23b-3p mimics (50 nM; cat. no. miR10000418-1-5; Guangzhou RiboBio Co., Ltd., Guangzhou, China), miR-23b-3p inhibitors (100 nM; cat. no. miR20000418-1-5; Guangzhou RiboBio Co., Ltd.) and scrambled miRNA mimic $(50 \mathrm{nM}$; cat. no. miR01201-1-5; Guangzhou RiboBio Co., Ltd.) were transfected into U2OS cells using Lipofectamine ${ }^{\circledR} 2000$ (Invitrogen; Thermo Fisher Scientific, Inc.) according to manufacturer's protocol. miR-23b-3p mimics, miR-23b-3p inhibitors and scrambled miRNA mimic were diluted with RPMI-1640 medium in the absence of FBS. Diluted Lipofectamine ${ }^{\circledR} 2000$ was then 

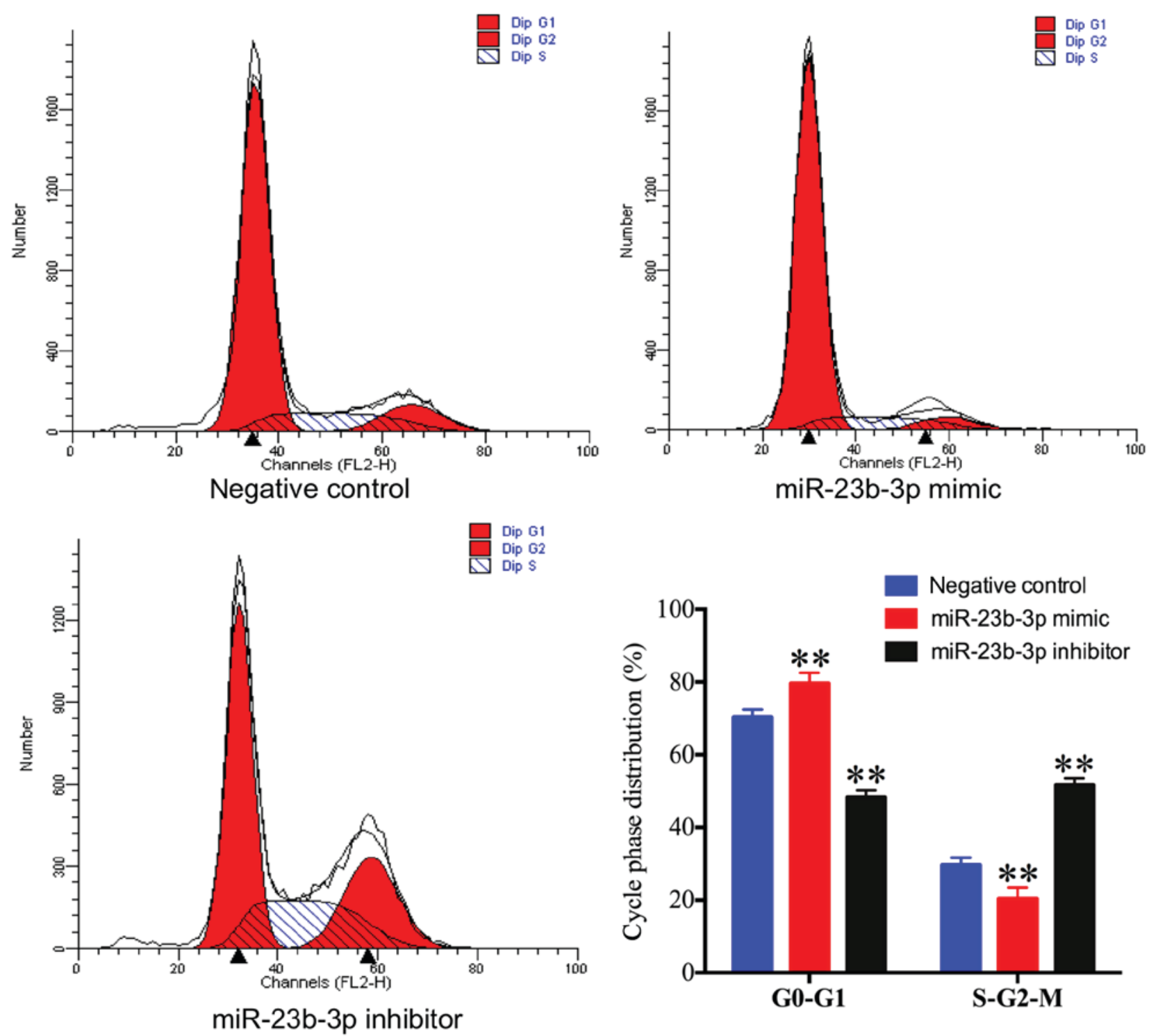

Figure 3. Effect of miR-23b-3p on U2OS cell cycle phase distribution. Representative flow cytometry images and comparisons of the cell cycle phase distribution among the different groups. The percentage of U2OS cell cycle phase distribution is expressed as the mean \pm standard deviation of three independent experiments. ${ }^{* *} \mathrm{P}<0.01$ vs. negative control. miR, microRNA.

separately incubated with miR-23b-3p mimics, miR-23b-3p inhibitors and scrambled miRNA mimic at room temperature for 20 min in order for the complexes to form. Following this, U2OS cells were incubated with the complex mixture at $37^{\circ} \mathrm{C}$ for $5 \mathrm{~h}$ in a humidified atmosphere of $5 \% \mathrm{CO}_{2}$. Finally, the culture medium was replaced with RPMI-1640 medium containing 10\% FBS and further incubated at $37^{\circ} \mathrm{C}$ for $48 \mathrm{~h}$.

CCK- 8 assays. Normal cultured U2OS cells $\left(1 \times 10^{5}\right)$ were inoculated into 96-well plates. Following cell adherence, according to the Lipofectamine ${ }^{\circledR} 2000$ transfection reagent protocol, the transfection of miR-23b-3p mimics, miR-23b-3p inhibitors and negative controls was performed. At 24, 48 and $72 \mathrm{~h}$ time intervals post-transfection, $10 \mu \mathrm{lCCK}-8$ solution (Beyotime Institute of Biotechnology) was added to each well, the plates were then incubated for $48 \mathrm{~h}$ at $37^{\circ} \mathrm{C}$. Optical density (OD) values at $450 \mathrm{~nm}$ were determined using an ELx808 absorbance microplate reader (BioTek Instruments, Inc., Winooski, VT, USA).
Flow cytometry analysis. Normal cultured U2OS cells $\left(1 \times 10^{5}\right)$ were inoculated into 96-well plates. Following transfection, the U2OS cells were fixed with $70 \%$ cold ethanol at $4^{\circ} \mathrm{C}$ for $18 \mathrm{~h}$ and then stained with propidium iodide $(30 \mu \mathrm{g} / \mathrm{ml})$ at $37^{\circ} \mathrm{C}$ for $30 \mathrm{~min}$. The percentage of U2OS cells in each phase of the cell cycle were determined using a BD FACSCalibur flow cytometer (BD Biosciences, Franklin Lakes, NJ, USA) and BD CellQuest ${ }^{\mathrm{TM}}$ Pro software version 5.1 (BD Biosciences). Following this, U2OS cells were digested via $0.25 \%$ trypsin, washed using PBS and then resuspended in $195 \mu 1$ of Annexin V-FITC binding buffer. Subsequently, Annexin V-FITC solution $(2.5 \mu \mathrm{g} / \mathrm{ml})$ was added and the solution was then incubated for $10 \mathrm{~min}$ at room temperature in the dark. Propidium iodide staining solution $(5 \mu \mathrm{g} / \mathrm{ml})$ was then added and the solution was incubated for $15 \mathrm{~min}$ at room temperature. Finally, the percentage of spontaneously apoptotic U2OS cells was determined using a BD FACSCalibur flow cytometer using BD CellQuest ${ }^{\mathrm{TM}}$ Pro software version 5.1 (BD Biosciences). 

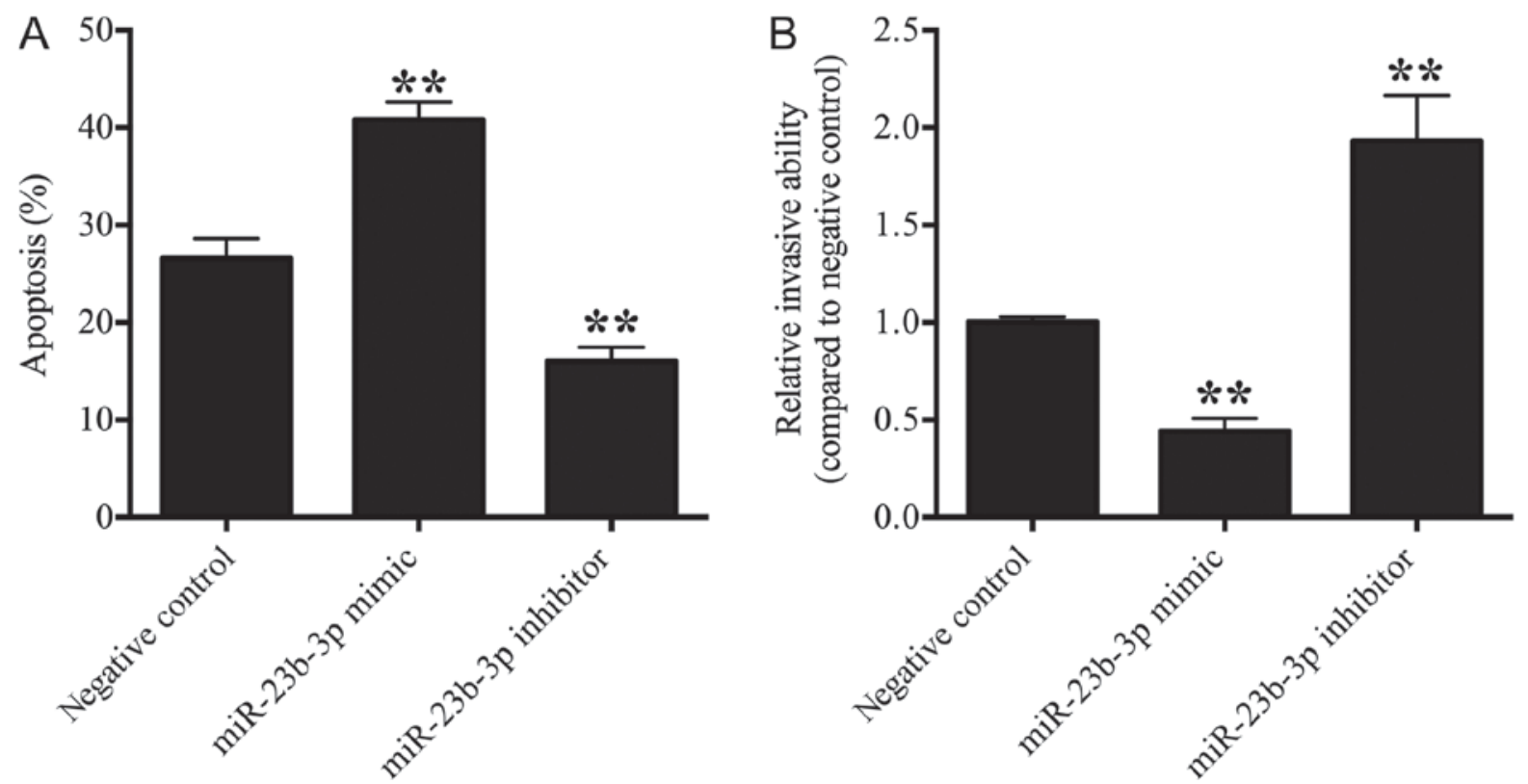

Figure 4. Analysis of the effects of miR-23b-3p on the (A) apoptosis and (B) invasive abilities of U2OS cells. ${ }^{* *} \mathrm{P}<0.01$ vs. negative control. Each experiment was repeated at least three times. miR, microRNA.

Transwell invasion analysis. The invasive capability of U2OS cells was investigated using Transwell invasion assays. miR-23b-3p mimics, miR-23b-3p inhibitors and negative controls were transfected into U2OS cells. RPMI-1640 medium (200 $\mu \mathrm{l}$ ) containing $1 \times 10^{3}$ U2OS cells was subsequently added to the Matrigel-coated, upper chamber (Corning Incorporated, Corning, NY, USA). The lower Transwell chamber was filled with $500 \mu 1$ of RPMI-1640 medium containing 10\% FBS. Following a $48 \mathrm{~h}$ incubation period, U2OS cells in the upper chamber were removed using a cotton swab. U2OS cells that invaded into the Matrigel and through the filter into the bottom of the transwell chamber were collected and stained with $100 \mu \mathrm{l}$ of $0.5 \mathrm{mg} / \mathrm{ml}$ MTT for $4 \mathrm{~h}$ at $37^{\circ} \mathrm{C}$. The reaction was terminated by adding $200 \mu \mathrm{l}$ /well dimethyl sulfoxide and absorbance at $570 \mathrm{~nm}$ was subsequently determined using an ELx808 absorbance microplate reader (BioTek Instruments, Inc.). All experiments were performed in triplicate.

Dual-luciferase reporter assay. In preliminary analyses, TargetScan tools (release 7.2; www.targetscan.org/) were used to predict the potential targets of miR-23b-3p, and the SIX1 3'-UTR was identified as a candidate region. Firstly, the 3'-untranslated region (UTR) fragment of the SIXI gene was amplified via PCR using genomic DNA isolated from hFOB1.19 cells, which was performed in accordance with the protocol outlined in the RT-qPCR subsection. Specific point mutations were then introduced into the 3'-UTR of the SIXI gene by overlap extension PCR cloning (41). Following this, PCR products were cloned into the pmiR-RB-Report ${ }^{\mathrm{TM}}$ reporter vector (Guangzhou RiboBio Co., Ltd.). A luciferase reporter plasmid (Guangzhou RiboBio Co.,Ltd.) was constructed, which included either the wild type (SIX1-W) 3'-UTR or mutated (SIX1-M) 3'-UTR. 293 cells $\left(3 \times 10^{5} / \mathrm{ml}\right)$ were inoculated into 6-well plates, and then the luciferase plasmid containing either SIX1-W 3'-UTR or SIX1-M 3'-UTR, and miR-23b-3p mimics or mimic negative controls (Genepharma Co., Ltd.), were cotransfected into 293 cells using Lipofectamine ${ }^{\circledR} 2000$. At $48 \mathrm{~h}$ post-transfection, 293 cells were collected and luciferase activity was determined using a luciferase assay kit (Promega Corporation, Madison, WI, USA) with a microplate reader. The reporter luciferase was normalized to Renilla luciferase activity.

Statistical analysis. All data are presented as the mean \pm standard error of the mean from at least three independent experiments. SPSS statistical software version 17.0 (SPSS Inc., Chicago, IL, USA) was used to perform statistical analyses. To determine statistically significant differences, the Student's t-test was used for comparisons between two groups, and one-way analysis of variance followed by Tukey's post hoc test was used for multiple comparisons. $\mathrm{P}<0.05$ was considered to indicate a statistically significant difference. GraphPad Prism software version 5.0 (GraphPad Software, Inc., La Jolla, CA, USA) was used to plot graphs.

\section{Results}

miR-23b-3p expression levels are downregulated in OS tissues and cells. The results of RT-qPCR analyses demonstrated that the expression of miR-23b-3p was significantly suppressed in OS tissues when compared with tumor-adjacent normal tissues $(\mathrm{P}<0.05$; Table I and Fig. 1A). However, the expression of miR-23b-3p in patients with OS was not associated with clinicopathological characteristics $(\mathrm{P}>0.05)$. The expression profile of miR-23b-3p in the three OS cell lines (MG-63, SaOS-2 and U2OS) was similar to that exhibited by OS tissues, and was significantly decreased when compared with the expression level observed in normal osteoblast hFOB1.19 cells (Fig. 1B).

miR-23b-3p suppresses $O S$ cell viability and proliferation. To investigate the effects of miR-23b-3p on OS cells, miR-23b-3p mimics, miR-23b-3p inhibitors and negative controls were 

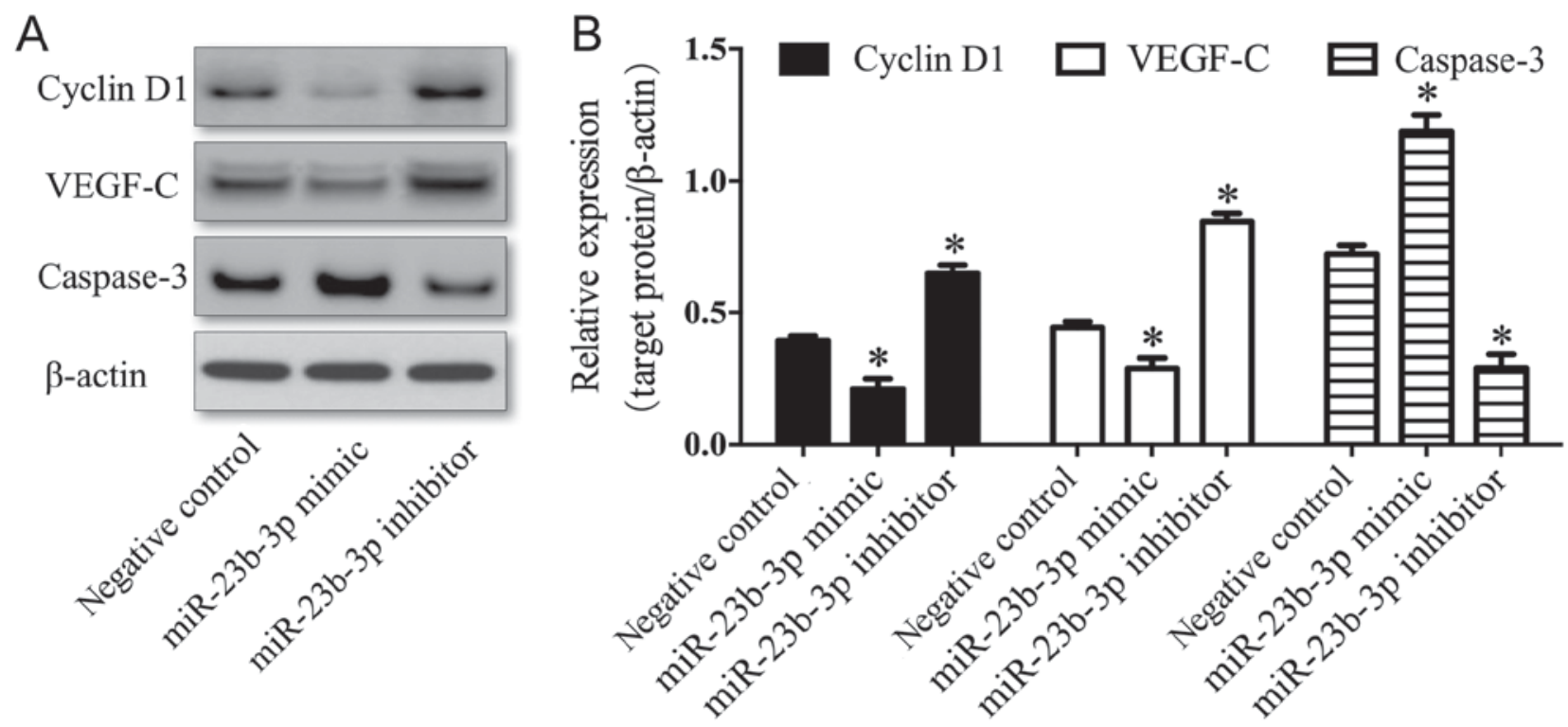

Figure 5. (A) Representative western blotting images for cyclin D1, caspase-3 and VEGF-C are presented. (B) Analyses comparing the expression levels of cyclin D1, caspase-3 and VEGF-C proteins among the different groups. " $\mathrm{P}<0.05$ vs. negative control. VEGF-C, vascular endothelial growth factor C; miR, microRNA.

transfected into U2OS cells, and the expression of miR-23b-3p was detected by RT-qPCR. The results demonstrated that the expression of miR-23b-3p was significantly suppressed in the miR-23b-3p inhibitor transfection group and significantly enhanced in the miR-23b-3p mimic transfection group when compared with the negative control group $(\mathrm{P}<0.01$; Fig. 2A), which confirmed that human OS U2OS cells, in which miR-23b-3p was either overexpressed or depleted, were successfully established.

The effect of miR-23b-3p on U2OS cell viability was determined using a CCK- 8 kit. The OD value was significantly decreased in the miR-23b-3p mimic transfection group and significantly increased in the miR-23b-3p inhibitor transfection group when compared with the negative control group $(\mathrm{P}<0.01$; Fig. $2 \mathrm{~B})$, which revealed that miR-23b-3p suppressed the cell viability of $\mathrm{U} 2 \mathrm{OS}$ cells.

The effect of miR-23b-3p on U2OS cell cycle phase distribution was investigated via flow cytometry. The percentage of U2OS cells in the S and G2-M phases were significantly decreased in the miR-23b-3p mimic transfection group and significantly increased in the miR-23b-3p inhibitor transfection group when compared with the negative control group (Fig. 3). By contrast, the percentage of U2OS cells at the G0-G1 phase was significantly increased in the miR-23b-3p mimic transfection group and significantly decreased in the miR-23b-3p inhibitor transfection group when compared with the negative control group (Fig. 3). These results demonstrated that miR-23b-3p inhibited OS cell cycle progression.

miR-23b-3p promotes OS cell apoptosis and suppresses $O S$ cell invasion. The effect of miR-23b-3p on the apoptosis of U2OS cells was detected by flow cytometry analysis. The number of apoptotic cells in the miR-23b-3p mimic transfection group was significantly increased $(\mathrm{P}<0.01)$, and number of apoptotic cells in the miR-23b-3p inhibitor transfection group was significantly decreased when compared with the

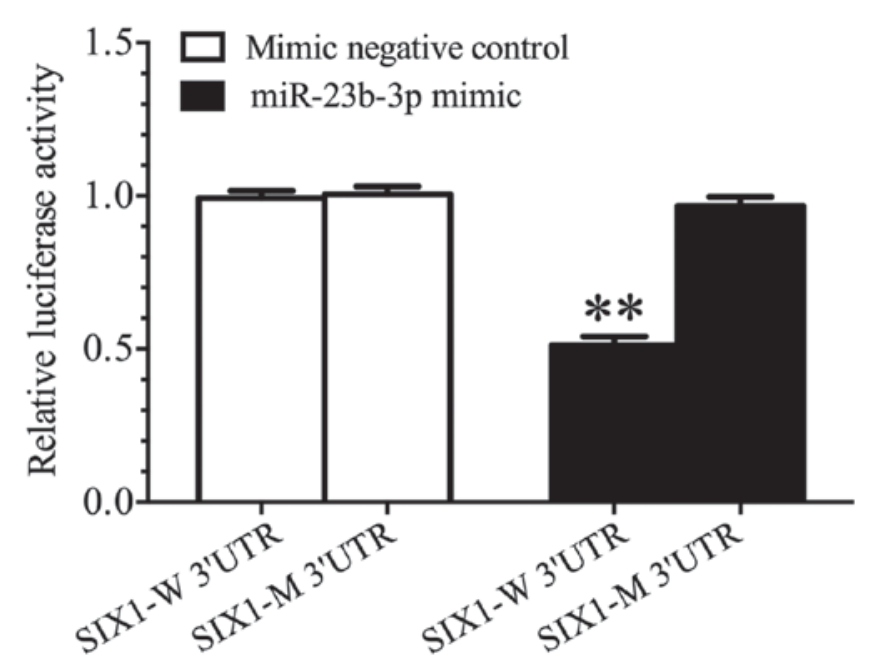

Figure 6. SIX1 represents a target of $\operatorname{miR}-23 b-3 p$, as determined by a dual-luciferase reporter assays. ${ }^{* *} \mathrm{P}<0.01 \mathrm{vs}$. corresponding mimic negative control groups. SIX1, sine oculis homeobox homolog 1; miR, microRNA; W, wildtype; M, mutant.

negative control group $(\mathrm{P}<0.01 ;$ Fig. $4 \mathrm{~A})$, which suggested that miR-23b-3p promoted OS cell apoptosis.

The effect of miR-23b-3p on the invasion of U2OS cells was detected via transwell invasion assays. The number of invasive cells was significantly suppressed in the miR-23b-3p mimic transfection group $(\mathrm{P}<0.01)$, and significantly increased in the miR-23b-3p inhibitor transfection group when compared with the negative control group $(\mathrm{P}<0.01$; Fig. 4B). These results indicated that miR-23b-3p suppressed the invasive ability of OS cells.

Effects of miR-23b-3p on the expression levels of cyclin DI, caspase-3 and VEGF-C in U2OS cells. To investigate the mechanisms underlying the effects of miR-23b-3p on the biological behavior of OS cells, the expression levels of cyclin 

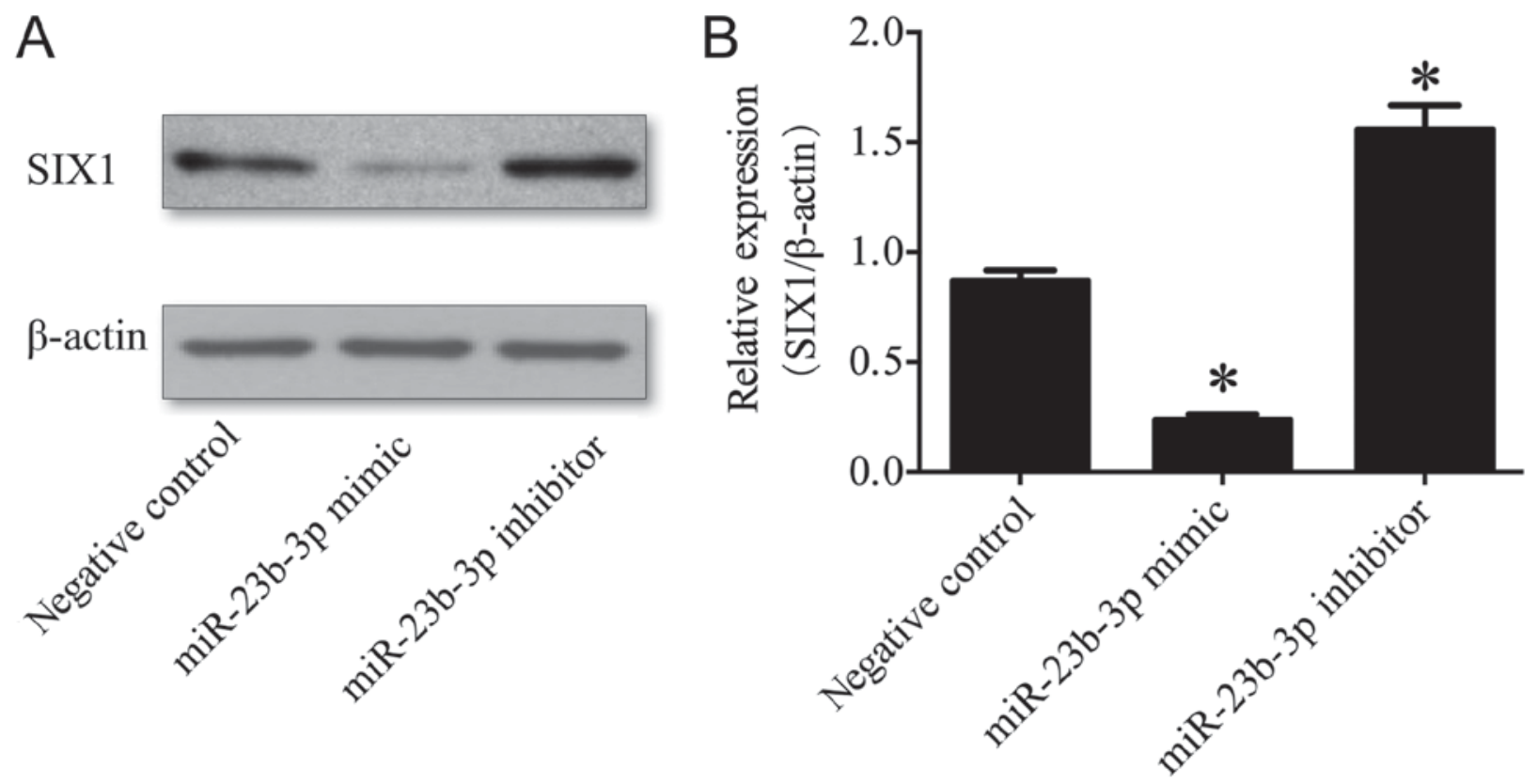

Figure 7. Effect of miR-23b-3p on the expression of SIX1 in osteosarcoma cells. (A) Representative western blotting images for SIX1 are presented. (B) The protein expression levels of SIX1 among the different groups. ${ }^{*} \mathrm{P}<0.05$ vs. negative control. SIX1, sine oculis homeobox homolog 1; miR, microRNA.

D1, caspase-3 and VEGF-C were determined by western blotting. The expression levels of cyclin D1 and VEGF-C were downregulated in the miR-23b-3p mimic transfection group $(\mathrm{P}<0.01)$, and significantly upregulated in the miR-23b-3p inhibitor transfection group, compared with the negative control group $(\mathrm{P}<0.01$; Fig. 5). By contrast, the expression of caspase-3 was upregulated in the miR-23b-3p mimic transfection group $(\mathrm{P}<0.01)$, and downregulated in the miR-23b-3p inhibitor transfection group, compared with the negative control group $(\mathrm{P}<0.01$; Fig. 5$)$. These results suggested that miR-23b-3p may be associated with decreased expression levels of cyclin D1 and VEGF-C, and increased expression levels of caspase-3, in U2OS cells.

SIX1 constitutes a direct target gene of $m i R-23 b-3 p$. In the present study, luciferase reporter gene analyses demonstrated that the activity of luciferase was significantly decreased in 293 cells co-transfected with the miR-23b-3p mimic and SIX1-W 3'-UTR when compared with cells co-transfected with either the mimic negative control and SIX1-W 3'-UTR, or the miR-23b-3p mimic and SIX1-M 3'-UTR ( $<<0.01$; Fig. 6). Our previous study revealed that the expression of SIXI mRNA in OS cell lines was significantly increased compared with normal human osteoblasts (2), which indicated that there was an inverse association between the expression of SIX1 and miR-23b-3p in OS. To investigate the role of miR-23b-3p in the regulation of SIX1 expression, western blotting was performed to determine the protein expression levels of SIX1 in U2OS cells transfected with either miR-23b-3p mimics or inhibitors. The results demonstrated that the expression levels of SIX1 protein were significantly suppressed in the miR-23b-3p mimic transfection group $(\mathrm{P}<0.01)$, and significantly enhanced in the miR-23b-3p inhibitor transfection group, compared with the negative control group $(\mathrm{P}<0.01$; Fig. 7), which demonstrated that miR-23b-3p may inhibit the expression of SIX1 protein in U2OS cells. Together, these results supported the hypothesis that $S I X 1$ represents a target gene of miR-23b-3p in OS cells.

\section{Discussion}

In the present study, the results of RT-qPCR analyses revealed that miR-23b-3p was significantly downregulated in OS tissues and cell lines when compared with tumor-adjacent normal tissues or normal osteoblast hFOB1.19 cells. The expression profile of miR-23b-3p in OS was similar to that exhibited in prostate cancer, cervical cancer and NSCLC (24-26), which suggested that miR-23b-3p may function as a tumor suppressor in OS. Despite the limited number of patients in the present study, the results indicated that the downregulation of miR-23b-3p may be associated with the tumorigenesis and progression of OS.

To further investigate the role of miR-23b-3p in OS, miR-23b-3p mimics, miR-23b-3p inhibitors and negative controls were transfected into U2OS cells to establish U2OS cells in which miR-23b-3p was either overexpressed or depleted. Using these lines, it was revealed that miR-23b-3p suppressed U2OS cell viability. The effects of miR-23b-3p on the U2OS cell cycle were determined via flow cytometry, and it was demonstrated that miR-23b-3p inhibited OS cell proliferation. These results were consistent with those of a previous study, which suggested that in human colon cancer, downregulated miR-23b-3p regulates either frizzled class receptor 7 or MAPK kinase kinase 1, subsequently mediating cancer proliferation as well as numerous stages of metastasis in vivo (42). Considering that cyclin D1 has been well established to represent an important regulator of cellular proliferation and tumorigenesis $(43,44)$, the expression of cyclin D1 in U2OS lines was determined in the present study. The results demonstrated that miR-23b-3p is involved in the 
suppression of cyclin D1 expression in U2OS cells, which suggested that miR-23b-3p may inhibit OS cell proliferation by suppressing the levels of cyclin D1. In addition, flow cytometry analyses demonstrated that miR-23b-3p also promotes OS cell apoptosis. Furthermore, a previous study revealed that miR-23b-3p promoted apoptosis and sensitized gastric cancer cells to chemotherapy (31), which indicated that miR-23b-3p may be applied for treatment for OS as well as the prediction of drug resistance, thus supporting the results of the present study. In addition, the results of the present study indicated that $\mathrm{miR}-23 \mathrm{~b}-3 \mathrm{p}$ may be associated with increased caspase-3 expression levels, which represents one of the most important biochemical markers of apoptosis (45) and a final effector of the apoptosis process (46), and thus may induce U2OS cell apoptosis.

Metastasis represents the leading cause of mortality in patients with OS (47). In the present study, the results of Transwell invasion assays revealed that miR-23b-3p suppressed the invasive ability of OS cells, which was consistent with previous findings in human colon cancer (42). Furthermore, in our previous study it was demonstrated that SIX1, a developmental transcription factor predicted to represent a potential target gene of miR-23b-3p, may enhance the expression of VEGF-C in OS (2). VEGF-C promotes cervical cancer cell invasion and migration (48), and enhances the metastatic ability of esophageal carcinoma (49), whereas knocking down VEGF-C suppresses gastric cancer cell migration (50). Therefore, VEGF-C was investigated in the present study, and the results demonstrated that miR-23b-3p is associated with decreased expression levels of VEGF-C in U2OS cells.

In addition, the results of our previous study revealed that SIX1 is upregulated in OS cell lines and that upregulated SIX1 may be associated with the promotion of the growth, proliferation and migration of U2OS cells, as well as the inhibition of U2OS cell apoptosis (2), which was in agreement with previous studies suggesting that enhanced SIX1 expression is associated with metastasis status or degree in breast cancer (35). Furthermore, our previous study also revealed that transfection with SIX1 increased cyclin D1 and VEGF-C expression levels, and decreased caspase-3 expression levels (2). In the present study, it was demonstrated that miR-23b-3p directly interacted with the SIX1 3'UTR and suppressed the expression of SIX1 protein in OS cells. Therefore, we hypothesized that miR-23b-3p downregulation may inversely regulate the expression of SIX1 in OS cells, thereby having an important role in the occurrence and development of OS.

The results of the present study demonstrated that miR-23b-3p was downregulated in OS, potentially because miR-23b-3p suppressed the proliferation and invasion abilities of OS cells, and enhanced OS cell apoptosis. In addition, the downregulated miR-23b-3p subsequently upregulated SIX1 expression, which may have induced the expression of cyclin D1 and VEGF-C, and inhibited the expression of caspase-3. In conclusion, these results suggested that OS exhibited downregulated miR-23b-3p and upregulated SIX1 expression levels, with the former inducing enhanced proliferation and invasion in OS cells, as well as the suppression of cell apoptosis via negative regulation of SIX1. Therefore, the miR-23b-3p/SIX1 pathway may represent a potential target for the treatment of OS.

\section{Acknowledgements}

Not applicable.

\section{Funding}

The present study was supported by the Nantong Science and Technology Innovation Program (grant no. YYZ17012).

\section{Availability of data and materials}

All data generated or analyzed during the present study are included in this published article.

\section{Authors' contributions}

HL, WW and XW designed the study. HL, WW, XW, XG, QC, ZP, XX and AW performed the experiments. HL, XG, QC, $\mathrm{ZP}$ and XX collected and assembled the data. HL and WW performed the data analysis. XW provided scientific expertise. HL wrote the manuscript.

\section{Ethics approval and consent to participate}

The present study was reviewed and approved by the Clinical Research Ethics Committee of the Haian Hospital of Traditional Chinese Medicine (Nantong, China), and written informed consent was obtained from all patients prior to enrollment.

\section{Patient consent for publication}

All patients agreed to publication and provided written informed consent.

\section{Competing interests}

The authors declare that they have no competing interests.

\section{References}

1. Moore DD and Luu HH: Osteosarcoma. Cancer Treat Res 162: 65-92, 2014.

2. Hua L, Fan L, Aichun W, Yongjin Z, Qingqing C and Xiaojian W: Inhibition of Six1 promotes apoptosis, suppresses proliferation, and migration of osteosarcoma cells. Tumour Biol 35: 1925-1931, 2014.

3. Jianwei Z, Fan L, Xiancheng L, Enzhong B, Shuai L and Can L: MicroRNA 181a improves proliferation and invasion, suppresses apoptosis of osteosarcoma cell. Tumour Biol 34: 3331-3337, 2013.

4. Hughes DP: Strategies for the targeted delivery of therapeutics for osteosarcoma. Expert Opin Drug Deliv 6: 1311-1321, 2009.

5. Gao Y, Feng Y, Shen JK, Lin M, Choy E, Cote GM, Harmon DC, Mankin HJ, Hornicek FJ and Duan Z: CD44 is a direct target of miR-199a-3p and contributes to aggressive progression in osteosarcoma. Sci Rep 5: 11365, 2015.

6. Zhou W, Hao M, Du X, Chen K, Wang G and Yang J: Advances in targeted therapy for osteosarcoma. Discov Med 17: 301-307, 2014.

7. Poletajew S, Fus L and Wasiutynski A: Current concepts on pathogenesis and biology of metastatic osteosarcoma tumors. Ortop Traumatol Rehabil 13: 537-545, 2011 (In English, Polish).

8. Hurley C, McCarville MB, Shulkin BL, Mao S, Wu J, Navid F, Daw NC, Pappo AS and Bishop MW: Comparison of (18) F-FDG-PET-CT and bone scintigraphy for evaluation of osseous metastases in newly diagnosed and recurrent osteosarcoma. Pediatr Blood Cancer 63: 1381-1386, 2016. 
9. Di Leva G, Garofalo M and Croce CM: MicroRNAs in cancer. Annu Rev Pathol 9: 287-314, 2014.

10. Fang Q, Xu T, Wu C, Zhou S and Sun H: Biotargets in neural regeneration. Biotarget 1: 6-10, 2017.

11. Wu Y and Jiang M: The revolution of lung cancer treatment: From vaccines, to immune checkpoint inhibitors, to chimeric antigen receptor T therapy. Biotarget: 1:7, 2017.

12. Ohtsuka M, Tanemura $\mathrm{M}$ and Akamatsu $\mathrm{H}$ : Long noncoding RNAs regulate malignant phenotypes in colorectal cancer. Biotarget 2: 4, 2018.

13. Acunzo M, Romano G, Wernicke D and Croce CM: MicroRNA and cancer-a brief overview. Adv biol Regul 57: 1-9, 2015.

14. Lin S, Pan L, Guo S, Wu J, Jin L, Wang JC and Wang S: Prognostic role of microRNA-181a/b in hematological malignancies: A meta-analysis. PLoS one 8: e59532, 2013.

15. Zhou Q, Gallagher R, Ufret-Vincenty R, Li X, Olson EN and Wang S: Regulation of angiogenesis and choroidal neovascularization by members of microRNA-23 27 24 clusters. Proc Natl Acad Sci U S A 108: 8287-8292, 2011.

16. Cho S, Wu CJ, Yasuda T, Cruz LO, Khan AA, Lin LL, Nguyen DT, Miller M, Lee HM, Kuo ML, et al: miR-23 approximately 27 approximately 24 clusters control effector $\mathrm{T}$ cell differentiation and function. J Exp Med 213: 235-249, 2016.

17. Di Y, Zhang D, Hu T and Li D: miR-23 regulate the pathogenesis of patients with coronary artery disease. Int J Clin Exp Med 8: 11759-11769, 2015

18. Wang Y, Liang H, Zhou G, Hu X, Liu Z, Jin F, Yu M, Sang J, Zhou Y, Fu Z, et al: HIC1 and miR-23 27 24 clusters form a double-negative feedback loop in breast cancer. Cell Death Differ 24: 421-432, 2017.

19. Noma D, Inamura K, Matsuura Y, Ninomiya H, Ichinose J, Nakao M, Mun M, Ishikawa Y and Okumura S: ALK-rearranged lung adenocarcinoma showing intra-bronchial protrusion: A case of actually peripheral origin with a rare spreading pattern. Biotarget 1: 15, 2017.

20. Cai S, Chen R, Li X, Cai Y, Ye Z, Li S, Li J, Huang H, Peng S, Wang J, et al: Downregulation of microRNA-23a suppresses prostate cancer metastasis by targeting the PAK6-LIMK1 signaling pathway. Oncotarget 6: 3904-3917, 2015.

21. He Y, Meng C, Shao Z, Wang H and Yang S: MiR-23a functions as a tumor suppressor in osteosarcoma. Cell Physiol Biochem 34: 1485-1496, 2014

22. Haier J, Strose A, Matuszcak C and Hummel R: miR clusters target cellular functional complexes by defining their degree of regulatory freedom. Cancer Metastasis Rev 35: 289-322, 2016.

23. Mercatelli N, Fittipaldi S, De Paola E, Dimauro I, Paronetto MP, Jackson MJ and Caporossi D: MiR-23-TrxR1 as a novel molecular axis in skeletal muscle differentiation. Sci Rep 7: 7219, 2017.

24. Aghaee-Bakhtiari SH, Arefian E, Naderi M, Noorbakhsh F, Nodouzi V, Asgari M, Fard-Esfahani P, Mahdian R and Soleimani M: MAPK and JAK/STAT pathways targeted by miR-23a and miR-23b in prostate cancer: Computational and in vitro approaches. Tumour Biol 36: 4203-4212, 2015.

25. Campos-Viguri GE, Jimenez-Wences H, Peralta-Zaragoza O, Torres-Altamirano G, Soto-Flores DG, Hernández-Sotelo D, Alarcón-Romero Ldel C, Jiménez-López MA, Illades-Aguiar B and Fernández-Tilapa G: miR-23b as a potential tumor suppressor and its regulation by DNA methylation in cervical cancer. Infect Agents Cancer 10: 42, 2015.

26. Begum S, Hayashi M, Ogawa T, Jabboure FJ, Brait M, Izumchenko E, Tabak S, Ahrendt SA, Westra WH, Koch W, et al: An integrated genome-wide approach to discover deregulated microRNAs in non-small cell lung cancer: Clinical significance of miR-23b-3p deregulation. Sci Rep 5: 13236, 2015.

27. Yan J, Jiang JY, Meng XN, Xiu YL and Zong ZH: MiR-23b targets cyclin G1 and suppresses ovarian cancer tumorigenesis and progression. J Exp Clin Cancer Res 35: 31, 2016.

28. An Y, Zhang Z, Shang Y, Jiang X, Dong J, Yu P, Nie Y and Zhao Q: miR-23b-3p regulates the chemoresistance of gastric cancer cells by targeting ATG12 and HMGB2. Cell Death Dis 6: e1766, 2015

29. Jin L, Wessely O, Marcusson EG, Ivan C, Calin GA and Alahari SK: Prooncogenic factors miR-23b and miR-27b are regulated by Her2/Neu, EGF, and TNF-alpha in breast cancer. Cancer Res 73: 2884-2896, 2013.

30. Bricaud $\mathrm{O}$ and Collazo A: The transcription factor six1 inhibits neuronal and promotes hair cell fate in the developing zebrafish (Danio rerio) inner ear. J Neurosci 26: 10438-10451, 2006.
31. Christensen KL, Patrick AN, McCoy EL and Ford HL: The six family of homeobox genes in development and cancer. Adv Cancer Res 101: 93-126, 2008.

32. Yu C, Zhang B, Li YL and Yu XR: SIX1 reduces the expression of PTEN via activating PI3K/AKT signal to promote cell proliferation and tumorigenesis in osteosarcoma. Biomed Pharmacother 105: 10-17, 2018

33. Li Z, Tian T, Hu X, Zhang X, Li L, Nan F, Chang Y, Wang X Sun Z, Lv F and Zhang M: Targeting Six1 by lentivirus-mediated RNA interference inhibits colorectal cancer cell growth and invasion. Int J Clin Exp Pathol 7: 631-639, 2014.

34. Iwanaga R, Wang CA, Micalizzi DS, Harrell JC, Jedlicka P, Sartorius CA, Kabos P, Farabaugh SM, Bradford AP and Ford HL: Expression of Six1 in luminal breast cancers predicts poor prognosis and promotes increases in tumor initiating cells by activation of extracellular signal-regulated kinase and transforming growth factor-beta signaling pathways. Breast Cancer Res 14: R100, 2012.

35. Reichenberger KJ, Coletta RD, Schulte AP, Varella-Garcia M and Ford HL: Gene amplification is a mechanism of Six1 overexpression in breast cancer. Cancer Res 65: 2668-2675, 2005.

36. Wang CA, Jedlicka P, Patrick AN, Micalizzi DS, Lemmer KC, Deitsch E, Casás-Selves M, Harrell JC and Ford HL: SIX1 induces lymphangiogenesis and metastasis via upregulation of VEGF-C in mouse models of breast cancer. J Clin Invest 122: 1895-1906, 2012

37. Gong YN, Wang X, Wang J, Yang Z, Li S, Yang J, Liu L, Lei X and Shao F: Chemical probing reveals insights into the signaling mechanism of inflammasome activation. Cell Res 20: 1289-1305, 2010.

38. Tsai PW, Shiah SG, Lin MT, Wu CW and Kuo ML: Up-regulation of vascular endothelial growth factor $\mathrm{C}$ in breast cancer cells by heregulin-beta 1 . A critical role of p38/nuclear factor-kappa B signaling pathway. J Biol Chem 278: 5750-5759, 2003.

39. Takaesu G: Two types of TRAF6-dependent TAK1 activation in the IL-1 signaling pathway. Biotarget 2:2, 2018.

40. Livak KJ and Schmittgen TD: Analysis of relative gene expression data using real-time quantitative PCR and the 2(-Delta Delta C(T)) method. Methods 25: 402-408, 2001

41. Bryksin A and Matsumura I: Overlap extension PCR cloning. Methods Mol Biol 1073: 31-42, 2013.

42. Zhang H, Hao Y, Yang J, Zhou Y, Li J, Yin S, Sun C, Ma M, Huang $\mathrm{Y}$ and $\mathrm{Xi} \mathrm{JJ}$ : Genome-wide functional screening of miR-23b as a pleiotropic modulator suppressing cancer metastasis. Nat Commun 2: 554, 2011.

43. Pysz MA, Hao F, Hizli AA, Lum MA, Swetzig WM, Black AR and Black JD: Differential regulation of cyclin D1 expression by protein kinase $\mathrm{C}$ alpha and signaling in intestinal epithelial cells. J Biol Chem 289: 22268-22283, 2014.

44. Wang H, Wang X, Archer TK, Zwaka TP and Cooney AJ: GCNF-dependent activation of cyclin D1 expression via repression of Mir302a during ESC differentiation. Stem Cells 32: $1527-1537,2014$

45. Vagner T, Mouravlev A and Young D: A novel bicistronic sensor vector for detecting caspase-3 activation. J Pharmacol Toxicol Methods 72: 11-18, 2015.

46. Wang HC, Zhang ZT, Huang JS, Zhang P, Xiong NA and Wang T: The contribution of Cdc2 in rotenone-induced G2/M arrest and caspase-3-dependent apoptosis (vol 53, pg 31, 2014). J Mol Neurosci 55: 812-813, 2015.

47. Mirabello L, Koster R, Moriarity BS, Spector LG, Meltzer PS, Gary J, Machiela MJ, Pankratz N, Panagiotou OA, Largaespada $\mathrm{D}$, et al: A genome-wide scan identifies variants in NFIB associated with metastasis in patients with osteosarcoma. Cancer Discov 5: 920-931,2015.

48. Chen H, Suo K, Cheng Y, Zheng B and Xu L: Vascular endothelial growth factor $\mathrm{C}$ enhances cervical cancer migration and invasion via activation of focal adhesion kinase. Gynecol Endocrinol 29: 20-24, 2013.

49. Su CM, Su YH, Chiu CF, Chang YW, Hong CC, Yu YH, Ho YS $\mathrm{Wu} \mathrm{CH}$, Yen CS and Su JL: Vascular endothelial growth factor-C upregulates cortactin and promotes metastasis of esophageal squamous cell carcinoma. Ann Surg Oncol 4(Suppl 21): S767-S775, 2014.

50. Lim J, Ryu JH, Kim EJ, Ham S and Kang D: Inhibition of vascular endothelial growth factor receptor 3 reduces migration of gastric cancer cells. Cancer Invest 33: 398-404, 2015. 